\title{
GPS Tracking System Using Car Charger
}

\author{
Kavish Atul Sanghvi", Prianka Manoj Mestry \\ Thakur Polytechnic, 90 Feet Road, Thakur Complex, Kandivali (East), Mumbai 400101, Maharastra, India
}

Copyright $\subset 2017$ by authors, all rights reserved. Authors agree that this article remains permanently open access under the terms of the Creative Commons Attribution License 4.0 International License

\begin{abstract}
GPS is Global Positioning System; a space-based satellite navigation system that provides location and time information in all weather conditions, anywhere on or near the Earth where there is an unobstructed line of sight to four or more GPS satellites. GPS tracking system is a software system which allows you to track the route from any source to destination. But GPS tracking system using car charger is a modify concept of tracking system using GPS. This concept is to get a current location of a moving car which is driven by a common man and its user friendly. GPS tracking system using car charger uses car charger as power source for the system. It also displays latitude and longitude along with map.
\end{abstract}

Keywords GPS - Global Positioning System, USB Universal Serial Bus, UART - Universal Asynchronous Receiver/Transceiver

\section{Introduction}

Our project i.e. GPS Tracking System using Car Charger is a type of GPS unit which is basically used for searching places, getting distance between two places and many other applications. But our project simply gives the location of the car with real time location (latitude and longitude) which is not so accurately received in GPS system present in our mobile phone or any other GPS system that is in build in a car. Thus, our project helps in getting latitude and longitude along with accurate location which is shown in map that is linked with Google Map.This project presents an automotive localization system using GPS. The system permits localization of the automobile. The current location can be seen on the tracking application. The hardware component of the project is a standalone standard GPS unit. It gets the supply of power by the car battery charger unit. GPS unit gets the location information from satellites in the form of latitude and longitude with the help of magnetic antenna. This information is then sent to the computer using USB to RS232 serial port connector.

The presented application is a low-cost solution for positioning the car and also very useful in case of car theft situations, for monitoring adolescent drivers by their parents as well as in car tracking system applications. The proposed solution can be used in other types of application, where the information needed is requested rarely and at irregular period of time (when requested).

- GPS is extremely easy to navigate as it tells you to the direction for each turns you take or you have to take to reach to your destination.

- GPS works in all-weather so you need not to worry of the climate as in other navigating devices.

- The GPS costs you very low in comparison other navigation systems.

- The most attractive feature of this system is its $100 \%$ coverage on the planet.

Benefits of car tracking:

- Vehicle tracking systems can help to reduce running costs by specifically targeting those who speed and waste fuel. By focusing upon these drivers, it is possible to not only reduce fuel and maintenance bills, but to also reduce insurance premiums.

- $\quad$ Some insurance companies will offer around a thirty percent discount to companies who implement a GPS vehicle tracking system. This is not only because it encourages safer driving, but also helps recovery if thefts do occur.

- Business owners can find their most productive employees and use this information to implement further training or even a system of bonuses to enhance staff members' work ethic.

\section{Related Work}

Imagine you are standing somewhere on Earth with three satellites in the sky above you. If you know how far away you are from satellite A, then you know you must be located somewhere on the red circle. If you do the same for satellites $\mathrm{B}$ and $\mathrm{C}$, you can work out your location by seeing where the three circles intersect. This is just what your GPS receiver does, although it uses overlapping spheres rather than circles. The more satellites there are above the horizon the more accurately your GPS unit can determine where you are. A GPS receiver "knows" the location of the satellites because 
that information is included in the transmitted Ephemeris data (see below). By estimating how far away a satellite is, the receiver also "knows" it is located somewhere on the surface of an imaginary sphere centred at the satellite. It then determines the sizes of several spheres, one for each satellite and therefore knows the receiver is located where these spheres intersect.

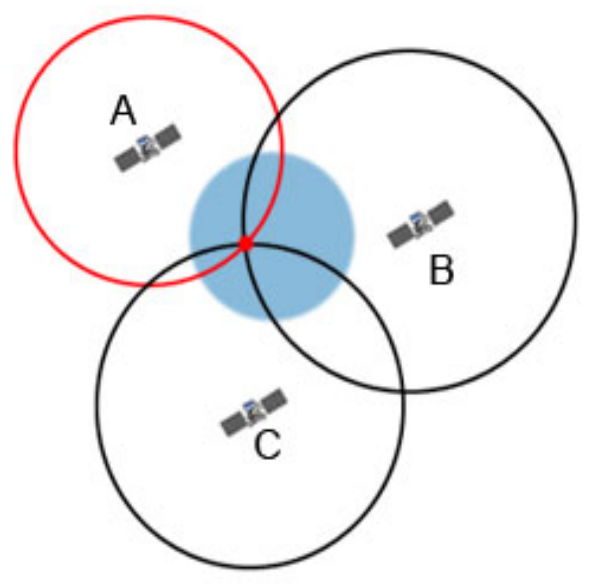

Figure 1. Range of satellite

\section{Architecture for Construction of GPS Tracking System}

Hardware - GPS Module, Magnetic GPS Antenna, USB to RS232 cable, Car charger, GPS Modem is used for receiving real time location (latitude and longitude) with the help of magnetic GPS antenna which is transferred to the application created in LabVIEW using USB to RS232 cable. Car charger is used to provide power supply to GPS modem for its internal use. The power given by car through car charger unit is been supplied to the GPS modem using car charger. The magnetic antenna gives the signal given by satellite to the GPS modem. The USB to RS232 connector is used to connect the modem to the laptop. The signals given by the antenna are processed by the modem and are given to the laptop in human readable form which is shown with the help of an interface created in LabVIEW software. It also shows location of the car on the Google map which is linked with our software made in LabVIEW.

\subsection{Phases Involved in GPU Module Construction}

Consider the following waterfall model which shows the list of sets that the GPS module construction has gone through:

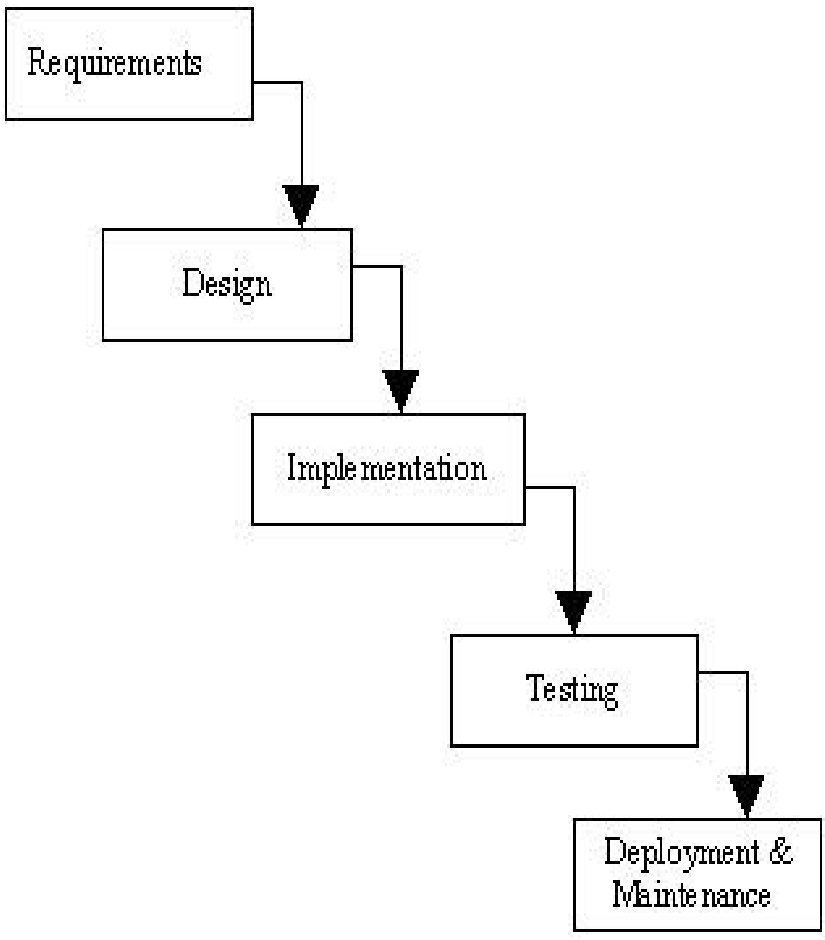

Figure 2. Waterfall model for GPS module construction

\subsubsection{Phase 1-Requirement Analysis}

The very first step to any development of software is the basic requirements to construct the project. The main components which are used in to construct GPS module are considered for requirement analysis. List of components are as follows:

\section{1) Hardware Requirements}

- GPS modem: The GPS modem is a device that accurately calculates geographical location by receiving information from GPS satellites.

- GPS Magnetic Antenna: Antenna is used to transfer the location signals received from GPS satellite to the GPS modem.

- 3G Dongle: $3 \mathrm{G}$ dongle provides $3 \mathrm{G}$ internet connection.

- USB to RS232 Cable: The cables provide a fast, simple way to connect devices with a RS232 level serial UART interface to USB.

- Car charger and Adapter: A car charger is a device that plugs into the 12-volt cigarette lighter outlet inside a vehicle, then connects to an electronic device such as a cell phone or MP3 player to charge the battery.

2) Software Requirements

- LabVIEW (short for Laboratory Virtual Instrument Engineering Workbench) is a system-design platform and development environment for a visual programming language from National Instruments. 
- The graphical language is named "G" (not to be confused with G-code). Originally released for the Apple Macintosh in1986, LabVIEW is commonly is used for data acquisition, instrument control, and industrial automation on a variety of platforms including Microsoft Windows, various versions of UNIX, Linux, and Mac OS X.

\subsubsection{Phase 2 -Design and Construction}

- The interface between software/application and hardware is a GPS modem

- The GPS modem acts as a mediator between hardware and software.

- It allows software to interact with hardware with the help of magnetic GPS antenna.

- The magnetic GPS antenna gives location (latitude and longitude) in the form of string, which is converted to floating point number with the help of a small program in LabVIEW.

- This floating-point number is then divided by 10000 to obtain executable or valid readings in latitude and longitude form separately.

- This conversion from string to floating point and then making the floating point into valid readings is done by a small program called "finalparser.vi".

- The valid latitude and longitude are then converted back to String location because GPS modem takes the readings in the form of String.

- The valid latitude and longitude are then converted back to String location because GPS modem takes the readings in the form of String.

- This URL is linked with Google Map so the real-time location is showed on map

\subsubsection{Phase 3- Implementation}

The project is made in such a way that it can be used in a moving car as early stated that its new concept is car charger. So we just need to have a complete setup in a car and have it running successfully. This project can be used anywhere around the world as it is globally interfaced software we have developed.

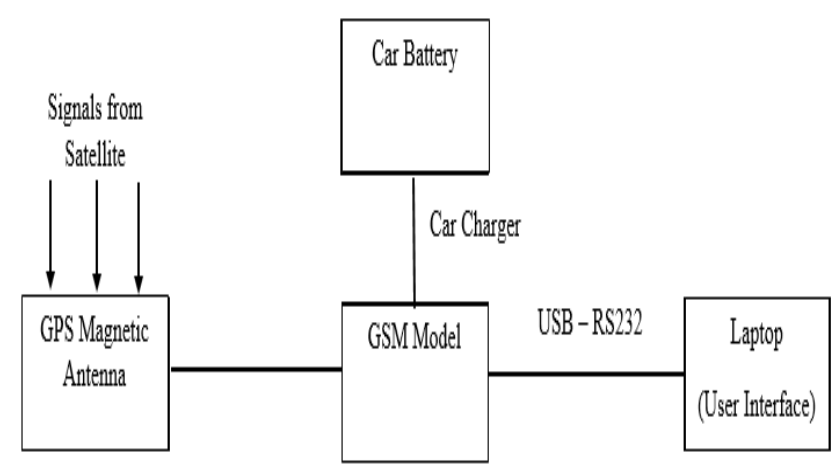

Figure 3. Block Diagram of GPS Tracking System

\subsubsection{Phase 4- Verification}

This phase is known as testing phase. It gives out the expected result of the project. The $100 \%$ success means the software which is developed has worked accordingly and it is working as per we have expected without any bugs or intervention of outside environment.

\subsubsection{Phase 5- Maintenance}

Components which are involved in and during this project development were maintained throughout the period of time i.e during first time execution, testing for few times so as to check whether its working everywhere also while presenting to higher authorities whenever was asked no device or components were damaged, they were preserved well.

\section{Future Scope}

- Accident detection is prevented.

- Vehicle tracking systems will vastly reduce your phone bills as it is no longer a necessity to constantly call employees to find their location. 


\section{Front and Back Panel}

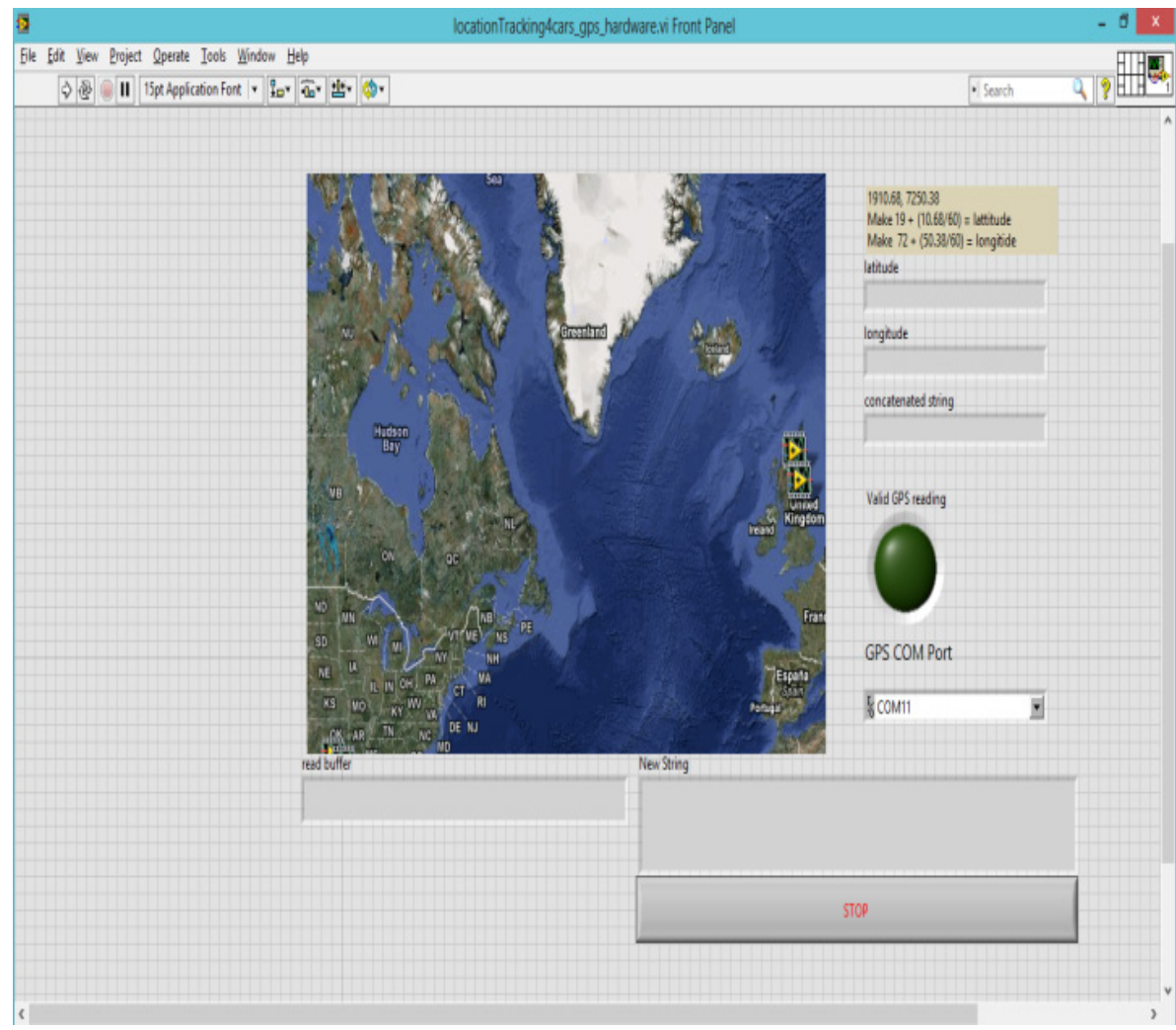

Figure 4. Front Panel of LabVIEW software

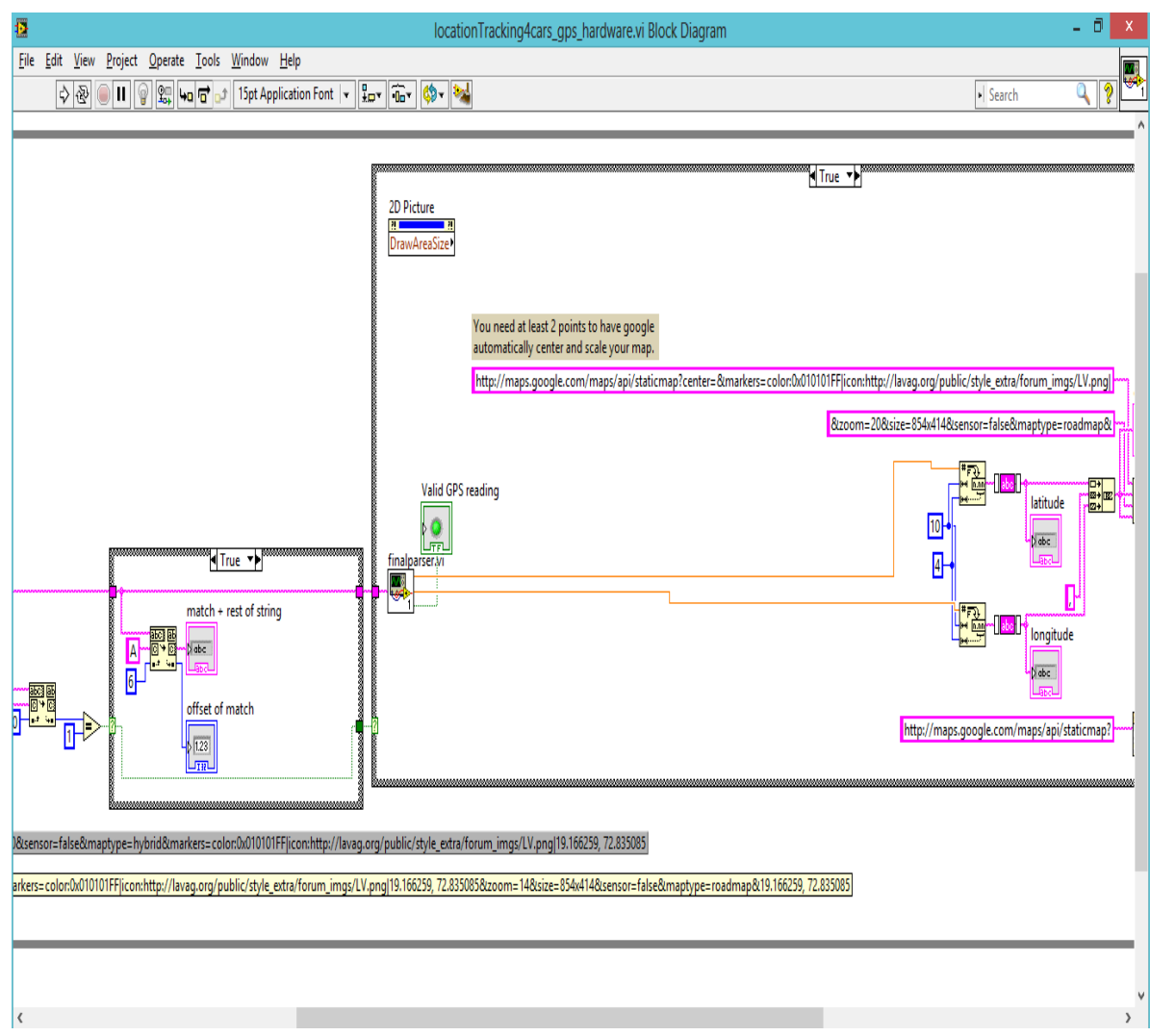

Figure 5. Back panel of LabVIEW Software 


\section{Acknowledgements}

Through this acknowledgement, we express our sincere gratitude to all those people who have been associated with our project and have helped us with it and make it a worthwhile experience. Firstly, we extend our thanks to our respected H.O.D. MRS. VAISHALI RANE, our entire Management and Computer Department and our project guide MS. PRIYANKA SAWANT who have shared their opinions and experiences through which we received the required information crucial for the report. We owe a great many thanks to all our staff for being supportive and helpful during the process of development of our project from documentation to application. Our deepest thanks to MS. PRIYANKA SAWANT, the guide of our project, for guiding and correcting our various documents with attention and care. We would also thank our institution THAKUR POLYTECHNIC and the principal Dr. S.M. GANECHARI and our faculty members without whom this project would have been a distant reality.

\section{REFERENCES}

[1] Introduction to GPS: The Global Positioning System

[2] Global Positioning System

[3] Latitude and Longitude Book by Rebecca Abert

[4] http://en.wikipedia.org/wiki/Global_Positioning_System

[5] http://www.gopalsystems.com/gps-gprs-solutions/gps-navigat ion/

[6] http://appinventor.mit.edu/explore/ai2/android-wheres-my-ca r.html

[7] http://www.slideshare.net/sumitvarshney50/gps-based-vehicl e-monitoring-system-copy

[8] https://www.theseus.fi/bitstream/handle/10024/65405/Thesis David_Davenne.pdf?sequence $=1$

[9] http://www.rei.com/learn/expert-advice/gps-reciever.htmlx 